BUILDING CODES AND SPECIFICATIONS

FOR THE ARAB WORLD 


\section{BUILDING CODES AND SPECIFICATIONS FOR THE ARAB WORLD}

Proceedings of a Seminar on Building Codes and Specifications, held in Amman, Jordan, 16-19 May 1982

\section{Edited by}

Fakhruddin Daghestani, Ruhi Sharif and Daud Jabaji

Seminar Sponsored by

The Royal Scientific Society

The Commission of the European Communities

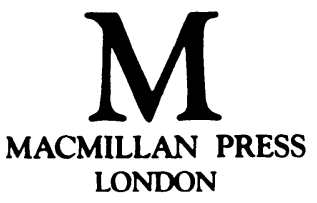


(c) The Royal Scientific Society, Amman, Jordan 1983

Softcover reprint of the hardcover 1st edition 1983 978-0-333-36826-8

All rights reserved. No part of this publication may be reproduced or transmitted, in any form or by any means, without permission.

First published 1983 by THE MACMILLAN PRESS LTD

London and Basingstoke

Companies and representatives

throughout the world

Typeset by

STYLESET LIMITED

Salisbury · Wiltshire

ISBN 978-1-349-07227-9

ISBN 978-1-349-07225-5 (eBook)

DOI 10.1007/978-1-349-07225-5 


\section{Contents}

Acknowledgements vii

Seminar Officers ix

List of Contributors $\quad \mathrm{xi}$

List of Participants xiii

Introduction $\quad 1$

F. A. Daghestani, R. Sharif, D. Jabaji

Keynote Address. A Unified Building Control System: Who Benefits and Who Controls the Controllers?

Andrew Short

ASMO Activities Related to Building Materials, Standards and the Arab Building Code

The General Secretariat of the Arab Organization for Standardization and Metrology (ASMO)

The Status of Building Specifications in the Arab World

Ruhi Sharif

International Harmonization of Building Regulations, Codes of Practice, and Standards

Günther Breitshaft

The New Building Specifications and Building Code of Jordan

Daud Jabaji

Building Research and Building Control

John Menzies

Problems Facing Consulting Firms Resulting from the Lack of Building Codes and Specifications in the Arab World

Tawfik Uwaydah 


\section{vi Contents}

Review of Practical Problems of Building Regulations and Supporting Documents Peter Bartle

Environmental Planning in Jordan: Experience and Prospects

Galeb Buqaien

The Role of Technical Evaluation and Certification

Sean Dunleavy

Organizing the Construction Industry in Jordan

Awni M. Saket

Review of Building and Civil Engineering Contract Systems and Legislation in the Member Countries of the European Community

Fernando Piccinini

The Amman Building Control Legislation

Ghassan Ghanem

The Belgium Experience in Procedures for Quality Certification of Building Components and in Environmental Planning and Regulations for Urban Areas

Henri Motteu

The Status of Specifications in Bahrain

Ismail Al-Shafei

The Practical Application of Building Specifications and Codes in the Middle East John Ashford

Some Aspects of the Proposed Iraqi Reinforced Concrete Code

Adnan Al-Ani, B. Jena 


\section{Acknowledgements}

This Seminar was prepared and held with the encouragement and support of many individuals. Our thanks are due to all those who contributed to its success.

Particular thanks are given to H.R.H. Crown Prince Hassan Ibn Talal for his encouragement and interest.

We also wish to thank Mr Awni Al-Masri, Minister of Public Works, Dr Albert Butros, Director General of the Royal Scientific Society, Mr Thomas O'Sullivan, Delegate of the Commission of the European Communities, and Dr Thafer Al-Sawaf, Secretary General of the Arab Organization for Standardization and Metrology, for providing their personal and institutional support for this Seminar.

Our thanks also go to Miss Nuha Hattar, Miss May Naber, Mr Farouk AlKhatib, and Mr Adib Zeidan for their assistance during the Seminar, and to the Macmillan Press for publishing this book.

Fakhruddin Daghestani

Ruhi Sharif

Daud Jabaji 


\section{Seminar Officers}

Chairman - First Session

Chairman - Second Session

Chairmen - Third Session

Chairman - Fourth Session
Dr Daud Jabaji

Head of Building Technology Division, Building Research Center, Royal Scientific Society, P.O. Box 6945, Amman, Jordan

Dr Thafer Al-Sawaf

Secretary General, Arab Organization for Standards and Metrology, P.O. Box 926161, Amman, Jordan

Dr Adnan Al-Ani

Building Research Centre, P.O. Box 127, AlGaderiah, Baghdad, Iraq

Mr Mukhtar Al-Shaibani

Head of Building and Construction Division, Saudi Arabian Standards Organization, P.O. Box 3437, Riyadh, Saudi Arabia

Mr Awni Saket

President, Contractors' Association.

Director General, National Company for Contracting and Engineering, P.O. Box 19170, Amman, Jordan 
x Seminar Officers

Chairmen - Fifth Session

Chairmen - Sixth Session

Seminar Coordinator

General Rapporteur
Dr Musa Resheidat

Acting Head, Department of CivilEngineering, Yarmouk University, Irbid, Jordan

Mr Nabih Bulos

Technical Advisor, Ministry of Culture and Youth, Amman, Jordan

Mr Lyth Shbeilat

President, Engineers' Association, P.O. Box 835, Amman, Jordan

Dr Fakhruddin Daghestani

Deputy Director General, Royal Scientific Society, P.O. Box 6945, Amman, Jordan

Dr Ruhi Sharif

Director, Building Research Center, Royal Scientific Society, P.O. Box 6945, Amman, Jordan

Dr Andrew Short

Honorary President, Euro-International Committee for Concrete (CEB), 70 Westminster Gardens, Marsham Street, S.W.1, London, United Kingdom 


\section{List of Contributors}

\section{Mr Ismail Al-Shafei}

Construction Manager, Technical Affairs Directorate, Ministry of Housing, P.O. Box 802, Bahrain

Dr Adnan Al-Ani

Building Research Centre, P.O. Box 127, Al-Gaderiah, Baghdad, Iraq

\section{Mr John Ashford}

Director and Chief Engineer, George Wimpey International Ltd, Hammersmith Grove, London W6 7EN, United Kingdom

Dr Adnan Awni

Assistant Secretary General, Arab Organization for Standardization and Metrology, P.O. Box 926161, Amman, Jordan

\section{Mr Peter Bartle}

Superintending Civil Engineer, Building Regulations Division, Department of the Environment, CID (BR) 7 Division, Room S13/12, 2 Marsham Street, London SW1P 3EP, United Kingdom

Mr Günter Breitschaft

Vizepräsident, Institut für Bautechnik, Reichpietschufer 72-76, D-1000 Berlin 30, West Germany

Mr Galeb Buqaien

Director General, Jordania Consult, P.O. Box 5070, Amman, Jordan

Mr Sean Dunleavy

Director, Construction Industry Division, Institute for Industrial Research and Standards (IIRS), Ballymun Road, Dublin 9, Ireland

Mr Ghassan Ghanem

Director of Studies Department, Municipality of Amman, Jordan

Dr Daud Jabaji

Head of Building Technology Division, Building Research Center, Royal Scientific Society, P.O. Box 6945, Amman, Jordan

Dr John Menzies

Assistant Director and Head, Geotechnics and Structures Department, Building Research Establishment, Garston, Watford, WD2 7JR, United Kingdom

Mr Henri Motteu

Head, Department R and D, Belgian Building Research Institute (C.S.T.C.), 41 rue du Lombard, 1000 Brussels, Belgium 


\section{Mr Hussein Musallam}

Building Supervisor, Engineering Division, KISR, Kuwait

\section{Mr Fernando Piccinini}

President of the Permanent Committee of the International Contractors' Section - Italian National Contractors' Association (A.N.C.E.), President of FERROCEMENTO, Via Guattani 16, 00161 Rome, Italy

\section{Mr Awni Saket}

Director General, National Company for Contracting and Engineering, Jabal Amman - 2nd Circle, Amman, Jordan

Dr Ruhi Sharif

Director, Building Research Center, Royal Scientific Society, P.O. Box 6945, Amman, Jordan

\section{Dr Andrew Short}

Honorary President, Euro-International Committee for Concrete (CEB), 70 Westminster Gardens, Marsham Street, S.W.1, London, United Kingdom

Mr M'hammed Slimani

Civil Engineer, Contrôle Technique de la Construction (CTC), Rue Kaddour Rahim Profongee, Hussein Dey, Algiers, Algeria

Mr Tawfik Uwaydah

Director, Department of Structural Engineering, Dar Al-Handasah Consultants, P.O. Box 7159, Beirut, Lebanon 


\section{List of Participants}

\section{Mr Nizar Aabidi}

Partner, Aabidi and Partners, P.O. Box 1547, Amman, Jordan

Mr Akram Abbasi

Chief Engineer, Sinimmar Engineering Co., P.O. Box 926116, Amman, Jordan Dr Mohammad Abdel-Halim

Department of Civil Engineering, Yarmouk University, Irbid, Jordan

Mr Koussai Abdel-Razzak

Technical Manager, Geotechnical Engineering and Materials Testing Co., P.O. Box 5382, Amman, Jordan

Mr Osama Abdull-Fattah

Civil Engineer, Saudi Arabian Standards Organization, P.O. Box 3437, Riyadh, Saudi Arabia

Dr Bassam Abu-Ghazaleh

Dean, Faculty of Engineering and Technology, University of Jordan, Amman, Jordan

Mr Akram Abu-Hamdan

Head of Architectural Section, Building Research Center, Royal Scientific Society, P.O. Box 6945, Amman, Jordan

Mr Mashari Abu-Saman

Electrical Engineer, Jordanian Electric Power Co. Ltd, P.O. Box 618, Amman, Jordan

Mr Mohammad Ajjour

Deputy Project Manager, United Group for Development and Contracting, P.O. Box 142, Officers Housing, Marg Al-Hamam, Jordan

Mr Khader Akkawi

Member of the Drafting Committee of the National Building Codes and Specifications, Building Research Center, Royal Scientific Society, P.O. Box 6945, Amman, Jordan

\section{Mr Hassan Akkour}

Member of the Drafting Committee of the National Building Codes and Specifications, Building Research Center, Royal Scientific Society, P.O. Box 6945, Amman, Jordan

Dr Adnan Al-Ani

Building Research Centre, P.O. Box 127, Al-Gaderiah, Baghdad, Iraq 
xiv List of Participants

\section{Mr Khalid Al-Haris}

Head of Laboratories, Cement Factories, P.O. Box 610, Amman, Jordan

Mr Emad Al-Khiari

Head of Specifications Division, Jordan Electricity Authority, P.O. Box 2310, Amman, Jordan

\section{Mr Ismail Al-Shafei}

Construction Manager, Technical Affairs Directorate, Ministry of Housing, P.O. Box 802, Bahrain

\section{Mr Mukhtar Al-Shaibani}

Head of Building and Construction Division, Saudi Arabian Standards Organization, P.O. Box 3437, Riyadh, Saudi Arabia

\section{Miss Salam Amarin}

Department of Specifications and Standards, Ministry of Industry and Trade, Amman, Jordan

\section{Mr John Ashford}

Director and Chief Engineer, George Wimpey International Ltd, Hammersmith Grove, London W6 7EN, United Kingdom

\section{Mr Shawki Attoum}

Industrial Chemistry Department, Royal Scientific Society, P.O. Box 6945, Amman, Jordan

Dr Adnan Awni

Assistant Secretary General, Arab Organization for Standardization and Metrology, P.O. Box 926161, Amman, Jordan

\section{Mr Khalid Azuqa}

Building Department, Ministry of Public Works, P.O. Box 1220, Amman, Jordan Miss Shadia Barakat

Head of Field Studies Division, Municipality of Amman, Amman, Jordan

\section{Mr Peter Bartle}

Superintending Civil Engineer, Building Regulations Division, Department of the Environment, CID (BR) 7 Division, Room S13/12, 2 Marsham Street, London SW1P 3EP, United Kingdom

\section{Mr Khalil Belbaisi}

Director of Building Department, Ministry of Municipal Affairs, Amman, Jordan Mr Günter Breitschaft

Vizepräsident, Institut für Bautechnik, Reichpietschufer 72-76, D-1000 Berlin 30, West Germany

\section{Mr Nigel Brewis}

Commission of European Communities, "Europe Center”, rue Archimede, B-1040 Bruxelles, Belgium

\section{Mr Nabih Bulos}

Technical Advisor, Ministry of Culture and Youth, Amman, Jordan Mr Galeb Buqaien

Director General, Jordania Consult, P.O. Box 5070, Amman, Jordan 
Dr Fakhruddin Daghestani

Deputy Director General, Royal Scientific Society, P.O. Box 6945, Amman, Jordan

\section{Mr William Dahdaleh}

Engineering Consultants Center, University of Jordan, Amman, Jordan

Mr Daud Dallal

Dar Al-Handasah Consultants, P.O. Box 2292, Amman, Jordan

Mr Mokhtar Daoudi

Contrôle Technique de la Construction (CTC), Rue Kaddour Rahim Profongee, Hussein Dey, Algiers, Algeria

\section{Mr Faris Daud}

Member of the Drafting Committee of the National Building Codes and Specifications, Building Research Center, Royal Scientific Society, P.O. Box 6945, Amman, Jordan

\section{Mr Talal Dibbes}

Member of the Drafting Committee of the National Building Codes and Specifications, Building Research Center, Royal Scientific Society, P.O. Box 6945, Amman, Jordan

\section{Mr Sean Dunleavy}

Director, Construction Industry Division, Institute for Industrial Research and Standards (IIRS), Ballymun Road, Dublin 9, Ireland

\section{Mr Main El-Hakim}

Architect, Building Research Center, Royal Scientific Society, P.O. Box 6945, Amman, Jordan

\section{Dr Zuhair El-Isa}

Assistant Professor, Faculty of Science, University of Jordan, Amman, Jordan

\section{Mr Ghassan Ghanem}

Director of Studies Department, Municipality of Amman, Amman, Jordan

Mr Fouad Gharaybeh

Civil Engineer, Royal Jordanian Air Force, Amman, Jordan

Mr Ibrahim Ghousheh

Owner-Designer of Supervising Office, Amman, Jordan

\section{Dr Osman Hamed}

Chemical Engineering Department, Faculty of Engineering and Technology, University of Jordan, Amman, Jordan

\section{Mr Hussam Hudhud}

Managing Director, Hudhud Shand Ltd, P.O. Box 926264, Amman, Jordan

Dr Nameer Hykal

Acting Head, Department of Architecture, Faculty of Engineering and Technology, University of Jordan, Amman, Jordan

Mr Sheikh Ismail

Arab Organization for Standardization and Metrology, P.O. Box 926161, Amman, Jordan 
Dr Daud Jabaji

Head of Building Technology Division, Building Research Center, Royal Scientific Society, P.O. Box 6945, Amman, Jordan

Mr Mohammad-Khair Kailani

Partner, Shubeilat Badran Kailani, Architects and Consultant Engineers, P.O. Box 182571, Amman, Jordan

Dr Mohammad-Basel Kathem

Assistant Professor, Department of Architecture, Faculty of Engineering and Technology, University of Jordan, Amman, Jordan

Dr I.E.D. Katkhuda

Head, Laboratories Division, Building Research Center, Royal Scientific Society, P.O. Box 6945, Amman, Jordan

Mr Khalil Khatib

National Resources Authority, P.O. Box 7, Amman, Jordan

Mr Senan Khliefat

Head, Sanitary Installation Division, Amman Water and Sewerage Authority, P.O. Box 2412, Amman, Jordan

Mr Hayel Khouri

Head, Concrete and Cement Unit, Building Research Center, Royal Scientific Society, P.O. Box 6945, Amman, Jordan

Mr Osama Madanat

Resident Engineer, Royal Corp of Engineers GHQ, P.O. Box 3235, Amman, Jordan

Dr Usama Madi

Assistant Professor, Faculty of Engineering and Technology, University of Jordan, Amman, Jordan

Dr John Menzies

Assistant Director and Head, Geotechnics and Structures Department, Building Research Establishment, Garston, Watford, WD2 7JR, United Kingdom

Mr Adel Miri'

Director, Civil Engineering Department, Jordan Electricity Authority, P.O. Box 2310, Amman, Jordan

\section{Mr Henri Motteu}

Head, Department $\mathrm{R}$ and D, Belgian Building Research Institute (C.S.T.C), 41 rue du Lombard, 1000 Brussels, Belgium

Mr Hussein Musallam

Building Supervisor, Engineering Division, KISR, Kuwait

Mr AbdulSalam Mustafa

Commander of Ready Mixed Concrete Group, Royal Corp of Engineers GHQ, P.O. Box 1663, Zerka, Jordan

Mr Nabih Nabhani

Economic Department, Royal Scientific Society, P.O. Box 6945, Amman, Jordan Mr Walid Nimry

Transport Planner, National Planning Council, P.O. Box 555, Amman, Jordan 


\section{Mr Taj-Ul-Deen Nuseibeh}

Occupational Safety and Health Advisor, Ministry of Labour, P.O. Box 8160, Amman, Jordan

\section{Dr Samih Qaqish}

Head of Civil Engineering Department, Faculty of Engineering and Technology, University of Jordan, Amman, Jordan

\section{Mr Munir Qaqish}

Head, Raw Materials Unit, Building Research Center, Royal Scientific Society, P.O. Box 6945, Amman, Jordan

\section{Dr Musa Resheidat}

Assistant Professor, Acting Head, Department of Civil Engineering, Yarmouk University, Irbid, Jordan

\section{Mr Awni Saket}

Director General, National Company for Contracting and Engineering, Jabal Amman - 2nd Circle, Amman, Jordan

Mr Kamel-Magdy Saleh

Member of the Drafting Committee of the Jordanian Building Code, Building Research Center, Royal Scientific Society, P.O. Box 6945, Amman, Jordan

\section{Dr Thafer Sawaf}

Secretary General, Arab Organization for Standardization and Metrology, P.O. Box 926161, Amman, Jordan

\section{Dr Ruhi Sharif}

Director, Building Research Center, Royal Scientific Society, P.O. Box 6945, Amman, Jordan

\section{Mr Ali Sharqawi}

Architect, Building Research Center, Royal Scientific Society, P.O. Box 6945, Amman, Jordan

Mr Mahmoud Shishani

Mechanical Engineer, Building Research Center, Royal Scientific Society, P.O. Box 6945, Amman, Jordan

\section{Dr Andrew Short}

Honorary President, Euro-International Committee for Concrete (CEB), 70 Westminster Gardens, Marsham Street, S.W.1, London, United Kingdom

\section{Mr M'hammed Slimani}

Civil Engineer, Contrôle Technique de la Construction (CTC), Rue Kaddour Rahim Profongee, Hussein Dey, Algiers, Algeria

\section{Mr Akram Sunna}

Under Secretary, Ministry of Public Works, P.O. Box 1220, Amman, Jordan

Dr Faisal Suyagh

Member of the Drafting Committee of the Jordanian Building Code, Building Research Center, Royal Scientific Society, P.O. Box 6945, Amman, Jordan Dr Magdy Tewfik

Assistant Professor of Urban and Regional Planning, Faculty of Engineering and Technology, University of Jordan, Amman, Jordan 
xviii List of Participants

Dr Ahmed Tobjy

Chemical Engineering Department, University of Jordan, Amman, Jordan

Mr Essam Toukan

Project Manager, Consulting Engineering Center, P.O. Box 8180, Amman, Jordan Mr Tawfik Uwaydah

Director, Department of Structural Engineering, Dar Al-Handasah Consultants, P.O: Box 7159, Beirut, Lebanon

Mr Najib Yaghmour

P.O. Box 921692, Amman, Jordan

Mr George Yannieh

Director of the Structural Department, Dar Al-Handasah Consultants, Beirut, Lebanon

Mr Khalid Yasin

Technical Manager, Mohamad A.R. Jardaneh Engineering Office, P.O. Box 9532. Amman, Jordan

Mr Bishr Zreiqat

Architect, Building Research Center, Royal Scientific Society, P.O. Box 6945, Amman, Jordan 